\title{
Data Collection Strategy in Low Duty Cycle Wireless Sensor Networks with Mobile Sink
}

\author{
Xiaofeng Tang1,2, Li Xie ${ }^{1,2}$ \\ ${ }^{1}$ State Key Laboratory of Novel Software Technology, Nanjing University, Nanjing, China \\ ${ }^{2}$ Department of Computer Science and Technology, Nanjing University, Nanjing, China \\ Email: tangxiaofeng@outlook.com, xieli@nju.edu.cn
}

How to cite this paper: Tang, X.F. and Xie, L. (2017) Data Collection Strategy in Low Duty Cycle Wireless Sensor Networks with Mobile Sink. Int. J. Communications, Network and System Sciences, 10, 227-239. https://doi.org/10.4236/ijcns.2017.105B023

Received: April 18, 2017

Accepted: May 23, 2017

Published: May 26, 2017

\begin{abstract}
The lifetime of wireless sensor networks can be improved by imposing low duty cycle, but doing so could not solve unbalanced energy consumption and will increase transmission latency. To avoid this, this paper gives a new method to collect data by mobile sink. The proper data collection route is selected according to the sink speed and buffer size of the sensors. The sensors only wake up when the sink approaches them. When certain sensors detect an emergency, the sink catches the message quickly and moves to the hotspot to decrease message relay in the network. The result of simulation by OPNET shows that this protocol can reduce transmission data in the network and prolong the network lifetime.
\end{abstract}

\section{Keywords}

Wireless Sensor Networks, Low Duty Cycle, Mobile Sink

\section{Introduction}

Wireless sensor networks (WSNs) have attracted a great deal of attention in both academia and industry in recent years. WSNs are composed of distributed autonomous sensors deployed in an unattended field or hostile area. Sensors monitor environmental conditions and transmit data to the sink node over multiple wireless hops. Because the sensors have limited energy reserves and are difficult to recharge, many researchers and developers have attempted to reduce sensor energy consumption and prolong network lifetime.

The many-to-one multi-hop traffic pattern in WSNs significantly increases the energy consumption of the nodes proximity to sink node. In effort to resolve problems such as energy hole and to balance energy consumption across the network to maximize lifetime, more sensors can be placed around sink node and the sensors scheduled to work alternately [1]. However, this increases the net- 
work cost and communication overhead. Mobile sinks or mobile relays can be used to solve energy hole problems, truncate the transmission route, and reduce the network's energy consumption [2] [3] [4].

Low duty cycle (LDC) protocol lets the node enter sleep mode when it is idle to reduce the energy consumption of the communication module [5]. However, it increases the latency of neighbor discovery and transmission delay. This has little effect on environmental monitoring and other routine tasks, but it renders the method unsuitable for real-time projects. For example, consider abnormal data detected by a forest fire monitoring system or mine gas monitoring system that must be transmitted to the observer in a timely manner. Researchers have proposed a variety of schemes to make the trade-off between transmission delay and node energy consumption. [6] presents a method to balance energy saving and end-to-end delay. [7] introduces a dynamic switch-based forwarding (DSF) mechanism that optimizes data delivery ratio, communication delay and energy consumption. Spatiotemporal delay control (SDC) [8] reduces communication delay by increasing duty cycle at individual nodes and optimizing the position of sink nodes. [9] establishes a sleep scheduling algorithm based on link-quality and energy-awareness to meet latency requirements and save nodes energy. [10] introduces opportunistic flooding with unreliable wireless links to reduce flooding delay and transmission energy. Though effective to some degree, none of these methods account for the data priority in regards to transmission. Reducing delay in routine tasks will not improve service performance, but instead will increase energy consumption.

Due to the long neighbor discovery time in LDC-WSNs, data accumulation is likely to occur when data bursts occur in the network. This causes network congestion resulting in data loss, increased transmission delay and reduced network lifetime. To avoid congestion, the route of the mobile sink can be adjusted for emergencies.

In this paper, we present a novel mobile sink routing strategy for routine tasks which can optimize the energy consumption of sensors and prolong network lifetime. When emergencies occur in the network, the proposed data diffusion technique is deployed to inform the sink of the event. The sink moves to a hotspot to reduce data transmission hops, which reduces the energy consumption of the whole network.

The rest of this paper is organized as follows: Section 2 describes the network model. Section 3 proposes the data collection strategy for routine tasks and the approach for emergencies is discussed in Section 4. Our simulation experiment and results are discussed in Section 5. Section 6 concludes this paper.

\section{Network Model}

We assume the following properties of the WSN deployed in a sensor field:

1) The sensor field is a circular area which has center $O$ and radius $R$ as shown in Figure 1. $N$ sensors

$S=\left\{s_{1}, s_{2}, \ldots, S_{n}\right\}$ are uniformly deployed in the area as density $\rho . S_{o}$ is the 


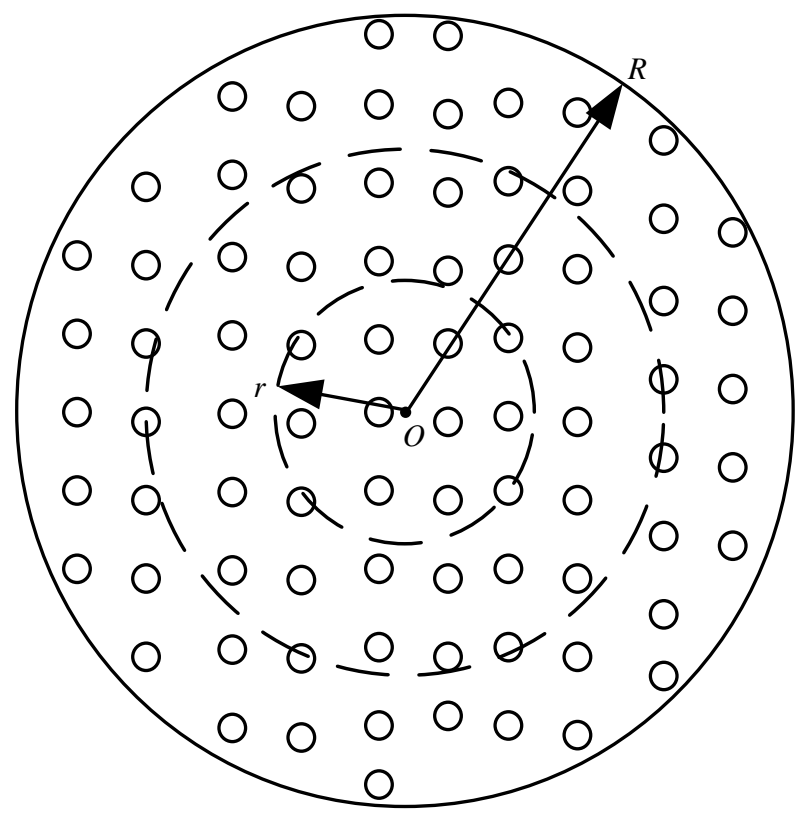

Figure 1. Monitoring area.

sink node.

2) Sensors have fixed positions. In order to save energy, they periodically wake up from sleep mode. The sink moves with speed $v$ and has sufficient energy; moving speed $v$ is much slower than the speed of data transmission.

3) Sensors collect data periodically. When detecting an emergency, their sampling rates increase to improve accuracy.

4) Sensors transmit data with max power in communication distance $r$. The energy consumption is independent with communication distance. Ignoring the energy consumption of storage and computation, the node energy consumption for communication is similar to which in [11].

$$
p=e\left(k_{r}+k_{t}\right)
$$

where $p$ is the energy consumption of one node in a sleep/wake cycle, $e$ is the energy consumption of one data packet transmission/receiving process, and $k_{t}, k_{r}$ are the quantities of data packets transmitted/received.

5) Data fusion is not considered. Sensors transmit raw data to the sink.

This paper uses the typical WSN setting as in [12]. In order to simplify the problem, sensors transmit data with fixed power. However, the method can also be applied to variable power and data fusion scenario.

WSNs have two working patterns: time-driven and event-driven. The timedriven mode is generally used to collect data periodically, while the event-driven mode is used to detect abnormal conditions in the sensor field. In this paper, we discuss a network with a combined time-driven and event-driven pattern which periodically transmits routine data to the sink and timely informs the sink of abnormal events

When network works in a routine pattern, optimizing the sink route in tandem with the sleep/wake cycle of the sensors can reduce the energy consumption 
of the whole network. When an emergency occurs in the network, it is necessary to also account for the transmission delay, energy consumption and transmission fairness.

\section{Data Collection Strategy}

The best way to effectively save energy is to move the sink to the sensors to communicate with them directly. In this scenario, sensors only need to transmit their own data. The transmission cycle is the interval in which the sink accesses all the sensors. During a routine task, the frequency at which the sink accesses the sensors is limited by their buffer size. When the buffer is sufficiently large, the sensor sleep/wake cycle is determined by the speed of the sink. In this case, the sensor wakes up to transmit its data only when sink approaches it. When the buffer is not sufficiently large, data transmission must be performed before the buffer is full. Accordingly, the sink cannot access all sensors directly in one sleep/wake cycle. Transmission delay in both of these situations is one sleep wake cycle.

In the former of the two scenarios described above, the sink moves along a fixed path. Sensors calculate the sink arriving time and plan the sleep/wake cycle. We use a moving method similar to [13]. One sensor is chosen randomly and its location serves as the starting point, then the sink traverses other sensors successively. For one possible route, the sink starts from the center of the sensor field and moves along half of the circle even-multiples of $r$ away from the starting point and back to the start along the other half of the circle, as shown in Figure 2.

For the latter situation, we assume that the buffer will be full in time $t$. During this period, the moving distance of the sink is $l=v^{*} t$. Sensors transmit data to

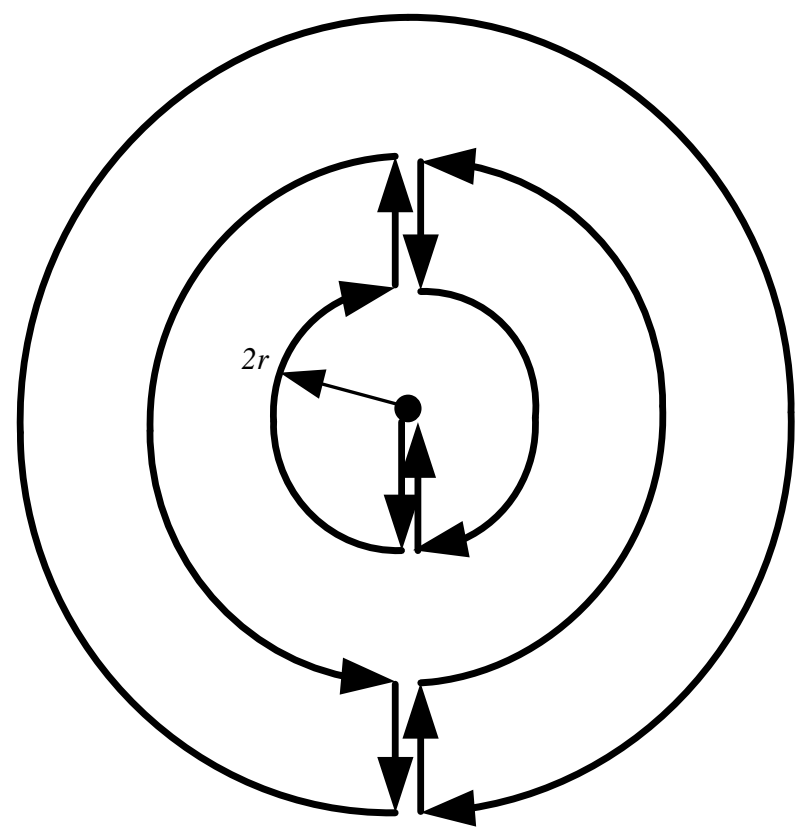

Figure 2. Sink moving route. 
sink only when the sink moves to the nearest point along the route. One sensor needs energy $p=e k_{g} h$ to transmit data in one cycle, where $e$ is the energy needed to transmit one single packet, $k_{g}$ is the number of packets collected by one sensor, and $h$ is hop count of the sensor from the sink. The energy consumption of the whole networks is

$$
p_{\text {total }}=\sum_{i=1}^{n} e k_{g} h_{i}=e k_{g} \sum_{i=1}^{n} h_{i}
$$

which indicates that minimizing the sum of the hop counts minimizing the total energy consumption.

When the sink is located at $O$, the number of sensors communicating with it directly is $\rho \pi r^{2}$. The outmost sensors in the field need $R / r$ hops to reach the sink and there are $\rho \pi R^{2}-\rho \pi(R-r)^{2}$ sensors in the outmost layer. The number of total hop counts is

$$
\begin{aligned}
H_{0}= & \rho \pi r^{2}+2\left[\rho \pi(2 r)^{2}-\rho \pi r^{2}\right]+\ldots \\
& +\frac{R}{r}\left[\rho \pi R^{2}-\rho \pi(R-r)^{2}\right] \\
= & \rho \pi\left[\frac{R}{r} R^{2}-(R-r)^{2}-(R-2 r)^{2}-\ldots\right. \\
& \left.-(2 r)^{2}-r^{2}\right]
\end{aligned}
$$

When the sink route is a circle, the maximum distance will be minimal. To move along this kind of route can balance energy consumption in the whole network. When the route is a circle with radius $m r, m=\{1,2, \ldots, R / r-1\}$, the total hop count is

$$
\begin{aligned}
H_{m}= & m \rho \pi r^{2}+(m-1)\left[\rho \pi(2 r)^{2}-\rho \pi r^{2}\right]+\ldots \\
& +\left\{\rho \pi(m r)^{2}-\rho \pi[(m-1) r]^{2}\right\} \\
& +\left\{\rho \pi[(m+1) r]^{2}-\rho \pi(m r)^{2}\right\}+\ldots \\
& +\left(\frac{R}{r}-m\right)\left[\rho \pi R^{2}-\rho \pi(R-r)^{2}\right] \\
= & \rho \pi\left[r^{2}+(2 r)^{2}+\ldots+(m r)^{2}\right] \\
& +\rho \pi\left\{\left(\frac{R}{r}-m\right) R^{2}-(R-r)^{2}-\ldots\right. \\
& \left.-[(m+1) r]^{2}-(m r)^{2}\right\}
\end{aligned}
$$

When $m=R / r$, Equation (4) can be rewritten as

$$
H_{R / r}=\rho \pi\left[\left(r^{2}+(2 r)^{2}+\ldots+(R-r)^{2}+R^{2}\right]\right.
$$

According to Equations (3)-(5), hop counts is minimum when

$$
m=\sqrt{\frac{3(R / r)^{2}-1}{6}}
$$

Thus, when $l \leq m r$, the sink should move along the circle with perimeter $l$ to optimize the energy consumption. When $l \geq m r$, the sink should move along the circle with perimeter $\mathrm{mr}$. In the latter case, the sink can also move along several small concentric circles while the sum of the circles perimeter is less than $l$. This is similar to $l>2 \pi R$. 
When $l>2 \pi R$, the sink can move along different circles to collect data in one sleep/wake cycle. Assuming the sink moves along the circles $C_{1}$ with radius $m_{1} r$ and $C_{2}$ with radius $m_{2} r$, then moving along inner circle $C_{1}$ is similar to the aforementioned situation $l \leq m r$ and the sink collects data from sensors within the circle with radius $\frac{\left(m_{1}+m_{2}\right)}{2} r$. From Equation (6),

$$
m_{1}=\sqrt{\frac{8 m_{2}^{2}}{49}-\frac{4}{21}}+\frac{m_{2}}{7}
$$

When the sink moves along outer circle $C_{2}$, the number of hop counts of sensors transmitting data to it is

$$
\begin{aligned}
H_{m_{2}}= & \rho \pi\left\{\left[\left(\frac{m_{1}+m_{2}}{2}+1\right) r\right]^{2}+\ldots+\left(m_{2} r\right)^{2}\right. \\
& \left.-\left(\frac{m_{2}-m_{1}}{2}\right)\left(\frac{m_{1}+m_{2}}{2} r\right)^{2}\right\} \\
& +\rho \pi\left\{\left(\frac{R}{r}-m_{2}\right) R^{2}-(R-r)^{2}-\ldots\right. \\
& \left.-\left[\left(m_{2}+1\right) r\right]^{2}-\left(m_{2} r\right)^{2}\right\}
\end{aligned}
$$

As indicated in Equation (6), when $R>4 r$, the sink moving along the circle with radius $0.7 R$ can achieve optimal energy consumption of the whole network and the circle divides the sensor field into two equal areas. Thus, assuming $C_{1}$ and $C_{2}$ divide their collecting area equally, the area of the ring between $C_{1}, C_{2}$, and the collecting area of route $C_{1}$ are both half of the sensor field

$$
\left\{\begin{array}{l}
\pi\left(m_{2} r\right)^{2}-\pi\left(m_{1} r\right)^{2}=\frac{1}{2} \pi R^{2} \\
\pi\left[\left(\frac{m_{1}+m_{2}}{2}\right) r\right]^{2}=2 \pi\left(m_{1} r\right)^{2}
\end{array}\right.
$$

Accordingly, $m_{1} \approx 0.46 R / r, m_{2} \approx 0.84 R / r$. These results satisfy Equation (7). By plugging the approximate results $m_{2}=(2 \sqrt{2}-1) m_{1}$ into Equations (4) and (8), the conditions of extremum $80 \sqrt{2} m_{1}^{2}-96 m_{1}^{2}=2(2 \sqrt{2}-1) r^{2}$ can be obtained, also, $m_{1} \approx 0.46 R / r$.

This can be extended to scenarios in which the sink moves along three or more circles. For convenience, we use LDC-COLLECT here to describe the moving method of the sink.

\section{Strategy for Emergency}

The sensors produce significantly more data when emergencies occur, under which circumstances the sensors' buffer will overflow if the sink moves along a fixed route. The observer will not obtain the entire data and fail to properly handle the event properly, which can lead to serious consequences.

\subsection{Strategy with Sufficient Energy}

In order to process emergencies in a timely manner, besides waking up and transmitting routine data periodically, sensors also need to wake up to exchange 
data with neighbors to spread hotspot messages in the network to inform the sink.

To send information in a straight line, sensors must be able to exchange data with all their neighbors-this is the quickest way to inform sink-but they must do so in strict time synchronization, which is difficult to achieve in large-scale networks. It is also not economical in regards to energy saving. In order to reduce the number of sensors evolving through data exchange, we set the sensors to transmit hotspot message to the center of the sensor field. These sensors then relay the message to the sink as shown in Figure 3.

There may be no sensors being deployed in the center of the sensor field. In the first round of routine data collection, the sink contacts the nearest sensor to the center according to received signal strength indication (RSSI). Several sensors are chosen as agents to wake up in turn to relay messages. The relay node does not know the location of the sink. It transmits message along different radii successively to find where the sink is located. After receiving the message, the sink replies a confirm message and forms a transmission path. Because the message spreads to the entire network in one sleep/wake cycle, the maximum delay of the build path is one sleep/wake cycle that is determined by the real-time requirements. Sensors wake up to exchange messages with neighbors in the same radius. When there are no events, the sensors go to sleep immediately. When catching an event message, the sensors stay awake to transmit it and wait for a reply from the sink. The sink can calculate the shortest path to events because all sensors receive event messages. And all sensors must calculate shortest path to the sink for routine data transmission, after which those sensors not related to relaying event data go to sleep to save energy until they have data to send. The sink moves to the event to reduce relaying data in the network. After an emergency, sink returns to its routine path.

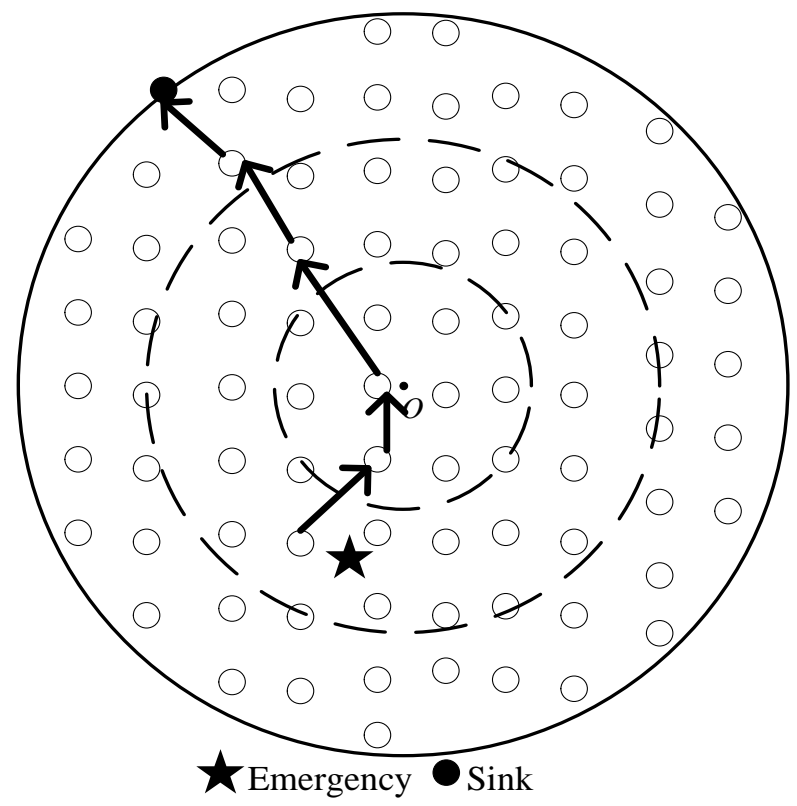

Figure 3. Event notification path. 
When more than one emergency occurs in the network, the sink must move to the convergence point of events data.

\subsection{Strategy with Insufficient Energy}

Sensors in the shortest path are tasked with relaying event data. To prolong the network lifetime, sensors with insufficient energy only wake up to transmit routine data and are not involved in emergency path construction.

When sensor energy decreases to a certain threshold, it informs upstream and downstream sensor in same radius and waits one-hop sensors to wake up to takeover data relay.

When sensors at the field center all have insufficient energy, they will not relay event messages. One-hop sensors to them are set as agent nodes which route event messages around the inner nodes. Similarly, when one-hop sensors lack sufficient energy, two-hop sensor will relay event message.

Algorithm 1. Strategy for Emergencies (EVENT-SOL)

1. Sensor $v_{e}$ detects event, generate $E V E \_M S G$, transmit it to $V_{n}$

IF there is upstream $v_{u}$, THEN $v_{n}=v_{u}$

ELSEIF there is downstream $v_{d}$, THEN $v_{n}=v_{d}$

ELSEIF wait for neighbor $V_{\text {neigh }}$ wake up, $v_{n}=V_{\text {neigh }}$

ELSE the sensor is isolated, network do not work

2. Transmit $E V E \_M S G$ hop-by-hop to agent node

3. Agent node transmits $E V E \_M S G$ to downstream node and neighbors in turn.

4. When sensor with $E V E \_M S G$ meets SINK, send $E V E \_M S G$ to it.

5. Sink send ACK back along the route of $E V E \_M S G$ to agent node and build shortest path from $v_{e}$ to SINK., sink simultaneously moves along the path.

6. If other event happens, sink moves to data aggregation point according to event location

7. Sensors knowing events build shortest path to sink.

8. After events, sink moves back to routine path

\section{Simulation}

We used OPNET to simulate the proposed strategy for routine and emergency data collection. To estimate network performance in terms of network life time, data delay and data delivery rate, we set the experimental environment described Table 1.

In response to a network emergency, we assume that the event needs to be transmitted to the sink within 20 seconds. Because the sensor needs one sleep/wake cycle to inform the agent sensor in the field center and the sink needs one more sleep/wake cycle to get event message from the agent. To examine a worst case scenario, the sleep/wake cycle is set as 10 seconds. When there are no events, the sensors do not need to exchange event messages. We set sensors to keep awake for 0.5 seconds in one sleep/wake cycle, the time slot is 0.5 seconds and one sleep/wake cycle has 20 slots. Sensors along the same radius should be synchronized during network deployment to wake up at the same time slot. 
Table 1. Experimental parameters.

\begin{tabular}{cc}
\hline Parameters & Value \\
\hline Sensor field radius & $100 \mathrm{~m}$ \\
Number of sensors & 500 \\
Communication range & $\leq 10 \mathrm{~m}$ \\
Sink speed & $\leq 1 \mathrm{~m} / \mathrm{s}$ \\
Sensor buffer size & 100 packets \\
\hline
\end{tabular}

\subsection{Effect of Data Collection Rate}

The sink's route is relevant to the time it takes for the sensor buffer to fill. The sensor data shows in Figure 4, where sensors collect data every 5 seconds, 10 seconds and 20 seconds. For 5 seconds data collection, the sink moves along one circle as described in Section 3. For 10 seconds collection, sink moves along two circles. And for 20 seconds collection, sink can access each sensor directly.

When the time interval of data collection is shorter, the sink can access relatively few sensors directly and more data must be relayed. The number of data packets transmitted in one cycle in each of these scenarios is shown in Figure 5.

\subsection{Energy Consumption in Routine Task}

When sensors are deployed in a circle area, the sink moving along the periphery of the network can optimize the overall energy consumption [14]. Figure 6 shows number of data packets transmitted when using optimum mobility with short path routing (SPR) in [14] and LDC-COLLECT. In [14], sensors do not wait for the sink to close in before transmitting data. This decreases transmission delay, but increases the relay data and demands that sensors wake up more frequently to consume more energy. As shown in Figure 6, because the sink moves along the same route, the number of data packets grows linear with the data collection cycle.

\subsection{Network Status in Emergencies}

We compare EVENT-SOL in energy consumption and transmission delay with opportunistic flooding in [10] for scenario where events are detected. During the simulation running time, we set one event occurs from the 200th second to the 400th second, two events occur from the 600th second to the 1000th second and three events from 1200th to 1600th second. We set sensors collect data every 20 seconds for routine task and 0.1 seconds for emergency. Every event is detected by four sensors around it and event locations are random. We ran the simulation five times and took the average number of packets. Figure 7 shows the number of packets transmitted in the network during the simulation. Relay data increased significantly when events occurred. The number of data packets relayed is effectively decreased by using EVENT-SOL in emergencies combined with LDC-COLLECT.

Figure 8 shows which sensor consumes the most energy in the network. Han 


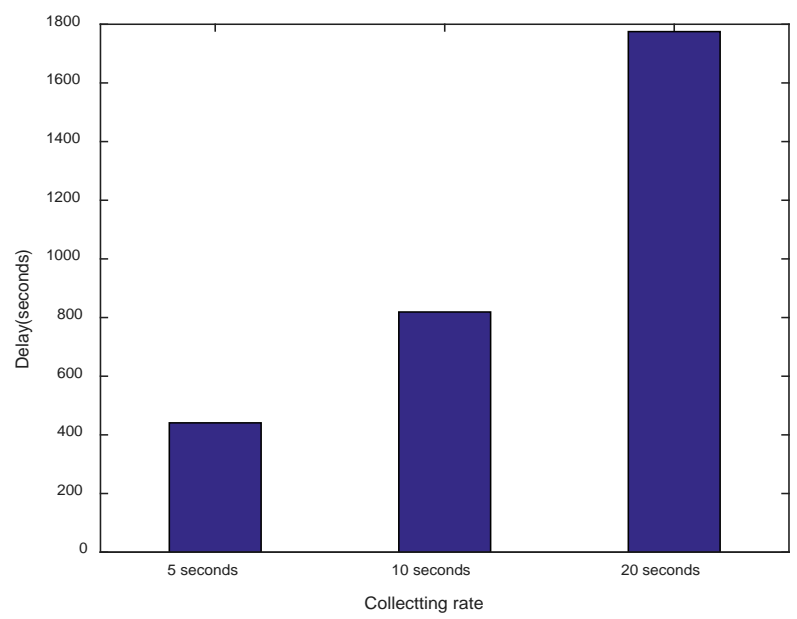

Figure 4. Collecting delay.

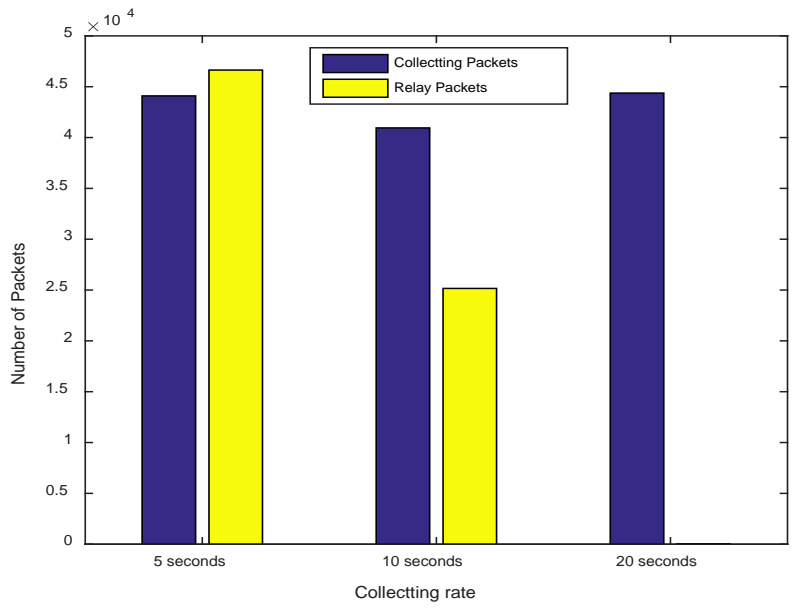

Figure 5. Packets in network.

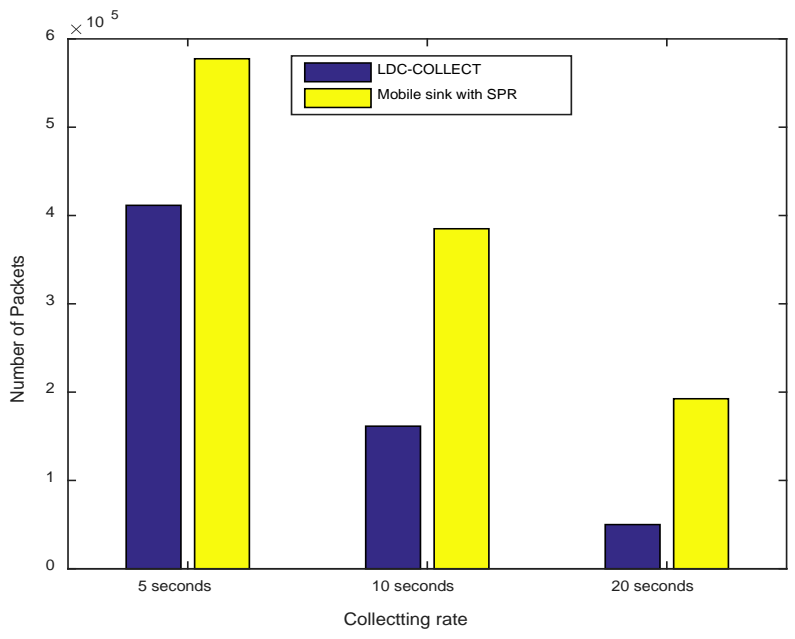

Figure 6. Packets collection compared with SPR.

dling emergencies requires more awake time to inform the sink and construct the path. The sensors in the transmission path must coordinate their working 


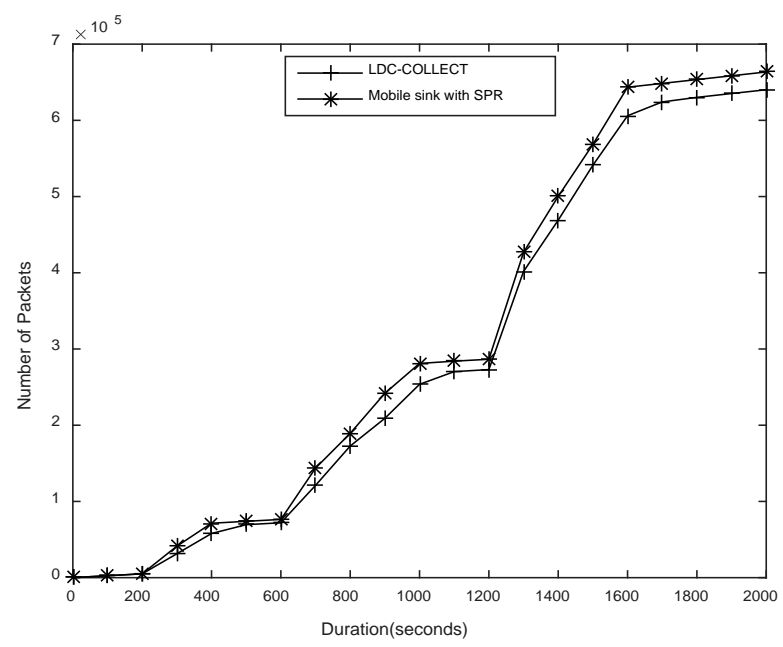

Figure 7. Packets transmitted in emergencies.

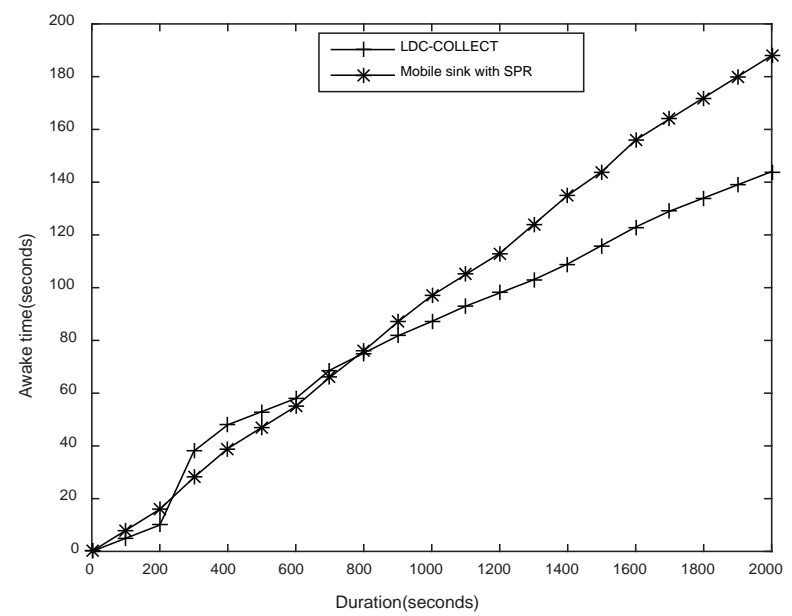

Figure 8. Sensor consuming most energy awake time.

slots for effective communication. In the simulation, the sensor consumed more energy by using opportunistic flooding than LDC-COLLECT in routine tasks and consumed less energy than EVENT-SOL in emergencies. As the event location changed in the network, the sensor consuming the most energy could be changed either. This balances energy consumption of the whole network.

In routine tasks, there is no transmission delay required. We simulated transmission delay only in emergencies. As shown in Figure 9, opportunistic flooding outperformed in regards to maximum delay. EVENT-SOL has to first report event and results in serious delay during this period. Increasing duty cycle can reduce the delay, but also reduce the network lifetime. EVENT-SOL can balance transmission delay and energy consumption.

\section{Conclusions}

This paper presents an energy-efficient data collection method applicable to LDC-WSNs. It consists of LDC-COLLECT for routine tasks and EVENT-SOL for emergencies. 


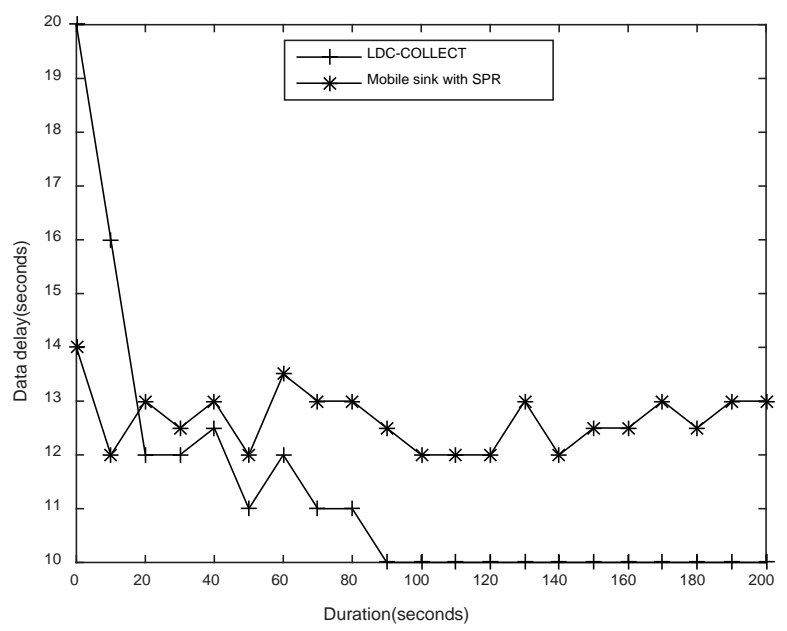

Figure 9. Data delay.

LDC-COLLECT arranges the sink to move along different routes according to the network size and collection frequency to save energy. EVENT-SOL reduces transmission delay and balances network load while prolonging the network lifetime.

The simulation results shows that the proposed method reduces sensors wake-up time in routine tasks and reduces energy consumption significantly. When encountering emergencies, it can be used to adjust network status and transmit events to the sink quickly.

\section{References}

[1] G.Grillo, Wu, X. and Chen, G. (2008) The Energy Hole Problem of Nonuniform Node Distribution in Wireless Sensor Networks. Chinese Journal of Computers, 31, 253-261. https://doi.org/10.3724/SP.J.1016.2008.00253

[2] Gandham, S.R., Daw, M., Prakash, R. and Venkatesan, S. (2003) Energy Efficient Schemes for Wireless Sensor Networks with Multiple Mobile Base Stations. Global Telecommunications Conference, 1, 377-381. https://doi.org/10.1109/GLOCOM.2003.1258265

[3] Luo, J. and Hubaux, J.-P. (2005) Joint Mobility and Routing for Lifetime Elongation in Wireless Sensor Networks. INFOCOM 2005. 24th Annual Joint Conference of the IEEE Computer and Communications Societies, 1735-1746.

[4] Wang, W., Srinivasan, V. and Chua, K.-C. (2005) Using Mobile Relays to Prolong the Lifetime of Wireless Sensor Networks. Proceeding of the 11 th Annual Int' I Conf. on Mobile Computing and Networking, 270-283. https://doi.org/10.1145/1080829.1080858

[5] Chen, L., Guo, S., Shu, Y., Zhang, F., Gu, Y., Chen, J., et al. (2011) Poster: Selective Reference Mechanism for Neighbor Discovery in Low-Duty-Cycle Wireless Sensor Networks. Proceedings of the 9 th ACM Conference on Embedded Networked Sensor Systems, 367-368. https://doi.org/10.1145/1995896.1995962

[6] Yang, X. and Vaidya, N. (2004) A Wakeup Scheme for Sensor Networks: Achieving Balance between Energy Saving and End-to-End Delay. 10th IEEE Real-Time and Embedded Technology and Applications Symposium, 19-26.

[7] Gu, Y. and He, T. (2007) Data Forwarding in Extremely Low Duty-Cycle Sensor Networks with Unreliable Communication Links. Proceedings of the 5 th Interna- 
tional Conference on Embedded Networked Sensor Systems, 321-334. https://doi.org/10.1145/1322263.1322294

[8] Gu, Y., He, T., Lin, M. and Xu, J. (2009) Spatiotemporal Delay Control for Low-Duty-Cycle Sensor Networks. 30th IEEE Real-Time Systems Symposium, 127-137. https://doi.org/10.1109/rtss.2009.12

[9] Chen, L., Wang, J., Zhang, J., Wang, Q., Liu Y., Yin, F, et al. (2014) Scheduling Scheme Algorithm in Low-Duty-Cycle WSN. Journal of Software, 25.

[10] Guo, S., He, L., Gu, Y., Jiang, B. and He, T. (2014) Opportunistic Flooding in Low-Duty-Cycle Wireless Sensor Networks with Unreliable Links. IEEE Trans-actions on Computers, 63, 2787-2802. https://doi.org/10.1109/TC.2013.142

[11] Gao, S., Zhang, H. and Das, S.K. (2009) Efficient Data Collection in Wireless Sensor Networks with Path-Constrained Mobile Sinks. IEEE Transactions on Mobile Computing, 10, 592-608. https://doi.org/10.1109/TMC.2010.193

[12] Heinzelman, W.R., Chandrakasan, A. and Balakrishnan, H. (2000) Energy-Efficient Communication Protocol for Wireless Microsensor Networks. Proceedings of the 33 rd Annual Hawaii International Conference on System Sciences, 2, 10. https://doi.org/10.1109/HICSS.2000.926982

[13] Xing, G., Wang, T., Xie, Z. and Jia, W. (2008) Rendezvous Planning in Wireless Sensor Networks with Mobile Elements. IEEE Transactions on Mobile Computing, 7, 1430-1443. https://doi.org/10.1109/TMC.2008.58

[14] Luo, J. and Hubaux, J.-P. (2005) Joint Mobility and Routing for Lifetime Elongation in Wireless Sensor Networks. Proceedings IEEE 24th Annual Joint Conference of the IEEE Computer and Communications Societies, 1735-1746.

\section{Submit or recommend next manuscript to SCIRP and we will provide best} service for you:

Accepting pre-submission inquiries through Email, Facebook, LinkedIn, Twitter, etc. A wide selection of journals (inclusive of 9 subjects, more than 200 journals)

Providing 24-hour high-quality service

User-friendly online submission system

Fair and swift peer-review system

Efficient typesetting and proofreading procedure

Display of the result of downloads and visits, as well as the number of cited articles

Maximum dissemination of your research work

Submit your manuscript at: http://papersubmission.scirp.org/

Or contactijcns@scirp.org 